\section{$\underset{\substack{\text { hommes } \\ \text { \& migrations }}}{ }$}

\section{Hommes \& migrations}

Revue française de référence sur les dynamiques

migratoires

$1301 \mid 2013$

Migrations et mondes ruraux

\title{
France-Hongrie, des liens secrets
}

\section{Hélène du Mazaubrun}

\section{OpenEdition \\ Journals}

\section{Édition électronique}

URL : http://journals.openedition.org/hommesmigrations/1937

DOI : 10.4000/hommesmigrations. 1937

ISSN : 2262-3353

\section{Éditeur}

Musée national de l'histoire de l'immigration

\section{Édition imprimée}

Date de publication : 1 janvier 2013

Pagination : 160-163

ISBN : 978-2-919040-21-6

ISSN : $1142-852 X$

\section{Référence électronique}

Hélène du Mazaubrun, «France-Hongrie, des liens secrets », Hommes \& migrations [En ligne], 1301 |

2013, mis en ligne le 29 mai 2013, consulté le 22 septembre 2020. URL : http://

journals.openedition.org/hommesmigrations/1937 ; DOI : https://doi.org/10.4000/

hommesmigrations. 1937

Ce document a été généré automatiquement le 22 septembre 2020.

Tous droits réservés 


\title{
France-Hongrie, des liens secrets
}

\author{
Hélène du Mazaubrun
}

1 Robert Futtersack est "tombé tout petit dans le goulasch, la marmite hongroise (plat traditionnel)" ". Né en France, marié à une Française, Robert Futtersack est bilingue franco-hongrois et cultive sa double culture. En témoignant dans le musée, il souhaite partager son héritage familial et son bagage culturel, et faire mieux comprendre la situation des immigrés des pays de l'Europe de l'Est.

"J'ai envoyé l'original de la pièce d'identité de ma mère comme don pour votre galerie. La carte de séjour de l'époque, c'est finalement assez banal. La plupart des étrangers étaient obligés de l'obtenir, c'est un document qui représentait leurs papiers. Pour trouver du travail par exemple, c'est une carte de séjour qu'il faut obligatoirement. Une carte qu'il fallait présenter à toute réquisition. C'est semblablement la même chose aujourd'hui, il faut un titre de séjour en tant qu'immigré pour pouvoir résider, travailler, vivre. Mais cette carte a une particularité, on y voit inscrit: 'Apatride ex-Tchécoslovaque, hongroise.' Pourquoi apatride? Pourquoi la France accorde ce statut aux immigrés qui sont dans le cas de mes parents? Qui est, il faut le rappeler, un cas rare."

\section{Devenir apatride}

3 À l'issue de la Première Guerre mondiale, les ressortissants de l'ancien royaume de Hongrie dissous doivent choisir la nationalité hongroise, tchécoslovaque ou yougoslave. Les traités de Versailles en 1919 et de Trianon en 1920 ont réorganisé les frontières de la Hongrie qui perdit $70 \%$ de son territoire. Un Hongrois sur trois vit alors en dehors des frontières de la Hongrie et doit choisir entre adopter la nationalité du nouveau pays ou conserver la nationalité hongroise, mais en étant considéré comme un "étranger dans son propre pays", devenu la Tchécoslovaquie, la Yougoslavie ou la Roumanie. "Ma mère, Therezia Béres, est née à Budapest en 1904, elle demeure hongroise, tandis que mon père, Maurice Futtersack, né à Eperjes-Prešov en 1901, opte pour la nationalité tchécoslovaque. Eperjes est le nom hongrois et Presov le nom slovaque qui reste le nom d'usage aujourd'hui. Mon père avait 20 ans à l'époque. Il a opté pour la nationalité des 'nouveaux', 'les vainqueurs', ceux qui ont imposé leur langue. Il aurait été étranger dans son propre pays s'il n'avait pas choisi la 
nationalité tchécoslovaque. En tant qu'étudiant en droit, il a fallu qu'il capitule. Une capitulation qui ne lui a pas beaucoup plu parce qu'il est venu en France. Ma mère, comme elle était de Budapest, rien n'a changé pour elle, elle est restée hongroise."

“Tous deux émigrent en France en 1924, mais ils ne se connaissent pas encore. Ma mère avait suivi son premier mari qui voulait améliorer son niveau de vie. Mon père cherchait du travail, n'ayant pas pu continuer ses études. En tant qu'immigrés, mes parents étaient quand même, il faut le dire, un peu perdus. Et ils devaient, pour vivre à tout prix, se faire de l'argent. Ils ont travaillé un peu partout. La France embauchait." Précisons que le nombre d'immigrés hongrois en France passe de 630 en 1921 à 19000 en 1931. Les Hongrois restent cependant une nationalité peu représentée (30000 aujourd'hui), ils sont très majoritairement installés à Paris. Maurice, comme de nombreux immigrés, subit le déclassement social. Il se retrouve à travailler dans une usine de pneus, bien que bachelier en langue allemande, puis étudiant en droit. "Ma mère, elle, faisait des ménages, la cuisine aussi. En fait, ils se débrouillaient. Et en même temps, ils devaient bien sûr apprendre le français. Donc leur problème était double, ils devaient parler le français non seulement pour pouvoir communiquer mais aussi pour s'assimiler et être assimilés. En fait, il est difficile pour moi de comprendre ou même de savoir vraiment pourquoi et comment mes parents ont émigré. En vérité je n'en sais rien. Je pense - ce sont des suppositions - qu'ils ont émigré pour des raisons économiques et peut-être par conviction. Mes parents se sont rencontrés dans les bals peu de temps après leur arrivée en France, dans les communautés hongroises, puis ils se sont mariés à Paris en 1936. Moi, je suis né en 1928.

Lorsque mes parents ont voulu régulariser leur situation après leur mariage, une contradiction apparaît. Devant fournir un certificat de nationalité, Therezia se voit indiquer par l'ambassade de Hongrie que, étant mariée à un Slovaque, elle a perdu sa nationalité hongroise. De son côté, l'ambassade de Tchécoslovaquie lui refuse la nationalité tchécoslovaque, son mariage avec mon père, Maurice, ne lui procurant pas automatiquement sa nationalité. Se retrouvant dans l'impossibilité de fournir un certificat de nationalité, ma mère reçoit le statut d'apatride' par l'administration française. La même année, nous retournons en Hongrie chercher ma demi-sœur, la fille de ma mère née de son premier mariage."

\section{Vivre sur la zone}

6 “À notre retour en France, quelques mois plus tard, on se retrouve avec d'autres immigrés sur 'la zone', tout autour du périphérique qui entoure Paris. Ce n'était certes pas des bidonvilles mais pas loin. Nous vivions comme des pauvres mais on vivait bien, on était quand même très heureux. Quand, plus tard, j'ai demandé à ma mère quel était l'endroit où elle avait été la plus heureuse, elle m'a répondu: 'La zone! Parce qu'à ce moment-là, j'étais avec mon mari et mes enfants."'

7 Lorsque la Seconde Guerre mondiale éclate, la situation se complique pour les immigrés. Enfant, Robert se souvient : “Quelques baraques se vidaient : les gens partaient en exode. Nous, les mômes, on ne savait pas où aller. Pas de famille à la campagne. [...] Ils ont bombardé les usines Renault à Billancourt, le jour du certificat d'études. On disait que les Allemands allaient fusiller tous les communistes et les brigades internationales. Nous, les mômes, on avait peur. À l'école, on disait rien, nous les sales étrangers. [...] C'était l'Occupation. Nos pères? On ne savait pas où ils étaient. Les Romanichels étaient repérés par leur couleur de peau. Nous, c'était par le nom de famille. Un matin, l'instituteur a dit: 'Votre camarade ne s'appelle plus Canaglia. Désormais, nous l'appellerons Brun.' Le veinard. Désormais, il n'avait plus à avoir 
peur. [...] Il a fallu se cacher. Les Allemands étaient tout près, dans les casernes. La zone se vidait. Chacun pour soi, comme il pouvait. On ne s'est même pas dit adieu, nous les mômes.

Mon père comme beaucoup d'immigrés à l'époque n'a pas eu d'autres possibilités que d'aller gagner sa vie en participant à la guerre d'Espagne en 1938. Les travailleurs français allaient soutenir, relayer les combattants républicains qui luttaient contre les franquistes et en échange un salaire était donné à la conjointe en France. Dès qu'il est arrivé là-bas, il a été emmené au front et il n'est jamais revenu. Voilà comment finit son histoire.

9 Ma mère, elle, c'est différent. À la Libération, nous avons eu la visite des Américains et ma sœur est tombée amoureuse d'un G.I., un soldat américain qui était enfant d'immigré hongrois aux États-Unis. Ils se sont mariés à Drancy et, un an après, elle se retrouvait aux États-Unis, donc elle émigre une deuxième fois. Et ma mère a voulu émigrer aux États-Unis avec moi, elle voulait qu'on parte là-bas pour rejoindre ma soeur. Mais moi, amoureux fou, je n'ai pas voulu y aller et elle est partie seule. Elle est devenue américaine au bout de cinq ans. Elle n'était plus du tout apatride, mais citoyenne américaine."

\section{Naître enfant d'immigrés}

"Je suis enfant d'immigrés. C'est un problème. Parce que, même si nous sommes nés en France, nous sommes également immigrés et nous avons subi toutes les conséquences de l'immigration. À l'école, j'étais un sale étranger et jusqu'au service militaire j'ai été traité de sale étranger. Je suis né en France, j'ai vécu en France, j'ai même grandi au sein de la culture française, j'étais le premier de la classe bien souvent, ça c'était ma petite revanche, mais j'étais malheureux. En France, on s'occupe trop des immigrés et pas assez des enfants des immigrés. Ces enfants n'ont rien à voir avec l'immigration, ils n'y sont pour rien, ils sont innocents dans toute cette affaire."

11 Robert Futtersack n'a pas immigré. Pourtant, il était important pour lui de reconstituer sa "petite Hongrie" chez lui : "On a en Hongrie une 'pièce propre', c'est-à-dire une pièce réservée dans la maison pour le jour où on a des invités. C'était une pièce dont le parquet était ciré. Il fallait marcher avec des patins dans la pièce. C'était ouvert aux invités qui venaient. Ça a disparu, les jeunes d'aujourd'hui ne font pas ça. La chambre propre, c'était la chambre où l'on exposait. Ça fait partie des coutumes, ça fait partie de ma personnalité." Robert y expose de l'artisanat hongrois : des sculptures en porcelaine, des gravures qui représentent les costumes traditionnels hongrois du berger, du noble, du voyageur, etc.

“Aujourd'hui je suis marié avec Jacqueline. Je lui serai toujours reconnaissant d'avoir bien voulu épouser un enfant d'immigrés. Nous avons quatre enfants, neuf petits-enfants et nous avons fêté, il y a peu, la naissance de notre quatrième arrière-petit-enfant. Je suis content aujourd'hui, mes enfants, eux, ils sont $100 \%$ français, ils n'ont aucun problème. On ne leur demandera pas la date de naissance de leur grand-mère!" Dans un tableau, Robert Futtersack a peint la France. À l'image du cadavre exquis, des trajectoires migratoires se poursuivent, y compris hors cadre: "Par ce tableau, j'ai voulu montrer que certains de nos compatriotes se considèrent comme 'émigrés' en région parisienne pour des raisons d'emploi. Ce sont les immigrés de l'intérieur." De multiples visages, jeunes et souriant, dessinent la France et portent des bannières sur lesquelles est inscrit : "Nous sommes tous des immigrés." 


\section{NOTES}

1. Le témoignage, retranscrit en partie dans cette chronique, correspond à un entretien réalisé le 24 janvier 2013 avec Robert Futtersack. 